\title{
Generation of pure single-photon states in commercial photonic-crystal fibers on telecommunication frequencies
}

\author{
A. A. Talipov ${ }^{1}$, A. G. Shmelev ${ }^{1,2}$, A. A. Shukhin ${ }^{2}$, I. Z. Latypov ${ }^{1,2}$ \\ ${ }^{1}$ Kazan Quantum Center, Kazan National Research Technical University after A. N. Tupolev-KAI, \\ 18 Chetaeva str., 420126 Kazan, Russia \\ ${ }^{2}$ Kazan E. K. Zavoisky Physical-Technical Institute, Sibirsky tract, 10/7, 420029 Kazan, Russia \\ bibidey@mail.ru
}

PACS 42.50.DV

DOI 10.17586/2220-8054-2017-8-3-347-350

\begin{abstract}
We studied the theoretical possibility of generating single-photon states in fibers with active profile dispersion formed by spontaneous four-wave mixing (SFWM) using femtosecond laser pulses. The possibility of eliminating the spectral correlation for single-photon states generated in commercial fibers was assessed. To model the structure of photonic crystal fibers and the modes of SFWM generation, an optimization was performed on the parameters of the pump and the fiber structure.
\end{abstract}

Keywords: single photon, spontaneous four-wave mixing, photonic crystal fiber, quantum optics.

Received: 17 April 2017

Revised: 25 April 2017

\section{Introduction}

A single-photon source is an essential part of many quantum information devices. The specific characteristics of such sources determine possibilities for their application [1]. Currently, the most promising trends are the following: 1) quantum memory devices and quantum calculation algorithms for searching in unordered databases; 2) quantum repeaters for building scalable quantum networks with high security; 3) applications for quantum metrology and quantum informatics.

Spontaneous parametric down-conversion (SPDC) and spontaneous four-wave mixing (SFWM) are currently the most promising and advanced methods to generate single photons. In fact, SPDC and SFWM methods imply that there is the possibility of obtaining a two-photon state of light. The main advantages of these methods include the following: high stability of obtained states in time; the potential to generate spectrally limited pulses at room temperature; the possibility to adjust optical wavelength and spectrum width as well as the minute influence of multi-photon states. Basic disadvantages of the methods include the random nature of single-photon generation, which leads to the probability of obtaining a two-photon state, and a correlation between signal and idler photons. The correlation is caused by idiosyncracies of the two-photon generation process. Thus, mixed single-photon states are obtained for SPDS and SFWM methods. This means that there is a spatial, polarization and spectral correlation between the pair of photons. The elimination of all these types of correlation is necessary to obtain a "clean" single-photon state. A fundamental difficulty is the elimination of the spectral correlation between the signal and idler photons. This can be overcome by the proper selection of parameters for the nonlinear medium and the laser's pump, such that the special matching conditions for the pump photon, signal and idler photons can be obtained. That is the essence of the problem for creating a single-photon source.

Single-mode structures have several advantages over other media: generation in single spatial mode; suppression of lateral spatial modes; and high consistency with fiber-optical networks. Photonic crystal fibers (PCF) have the potential to provide the conditions with spectrally limited laser pulses $[1,2]$. It should also be noted that the efficiency of single-photon states is strongly dependent upon the parameters of the nonlinear medium and pump pulses.

This article provides the analysis of clean single-photon generation based on the SFWM method and femtosecond spectrally scanning laser for the commercial PCFs.

\section{PCF model development}

In order to determine spectrum and correlation characteristics for the SFWM photon fields, it is necessary to determine exact values for the index of refraction and dispersion of PCFs at its length, which takes part in the parametric processes. Usually it is not possible to obtain an analytical solution for the complex internal structure of PCFs to determine its characteristics and its fundamental modes. This is why computations of the effective 
refraction index, dispersion and other characteristics of PCF are provided by means of numerical solution of Maxwell's equations. In this study, simulations were performed using Comsol Multiphysics software.

The obtained model of PCF hexagonal structure (core diameter $2.4 \pm 0.1 \mu \mathrm{m}$; distance between holes $2.9 \pm$ $0.1 \mu \mathrm{m}$; hollow region diameter $27 \pm 0.5 \mu \mathrm{m}$ ) corresponds to commercial fibers with zero dispersion at $800 \mathrm{~nm}$.

The diameter of holes $d$, the distance between them $p$ as well as core diameter are defined in the process PCF design. The type and applicability of the PCF determines the geometry of its microstructure. For example, fibers with high level of nonlinearity are promising for the generation of photon pairs and characterized by hexagonal structure with small sized core diameter. The core material for the model fibers is silica; holes are filled with air under atmospheric pressure. Searching for fundamental modes in the fiber core with distribution close to that of a Gaussian one is carried out for each wavelength within the modelling process. By changing the wavelength within certain range, it is possible to analyze the character of the following desired values: effective refraction index, path loss, dispersion and $c$.

Calculations for the effective index of refraction and dispersion as a function of wavelength are shown in Fig. 1 and Fig. 2. The effective index of refraction for a nonlinear medium decreases with increasing of wavelength and the ratio of holes diameter to distance between holes. Increasing the air filling ratio forms a fundamental mode that occurs in the fiber core. Monitoring the parameters of fiber cladding and its microstructure, in general, allows one to form an emission into the fiber core without significant losses. For the model of the fibers, dispersion attains a negative value over the range $600-800 \mathrm{~nm}$, equals zero from $800-900 \mathrm{~nm}$ and has positive values for higher wavelengths. Evaluation of dispersion curves allows one to determine wavelength range with zero dispersion as well as the generation and propagation conditions for the signal and idler photons.

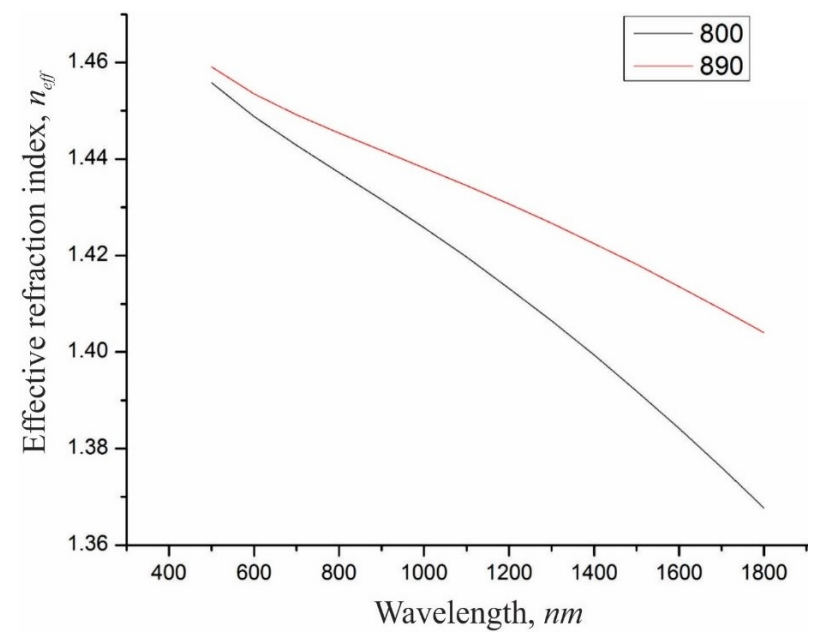

FIG. 1. Effective refraction index $n_{\text {eff }}$ as a function wavelength

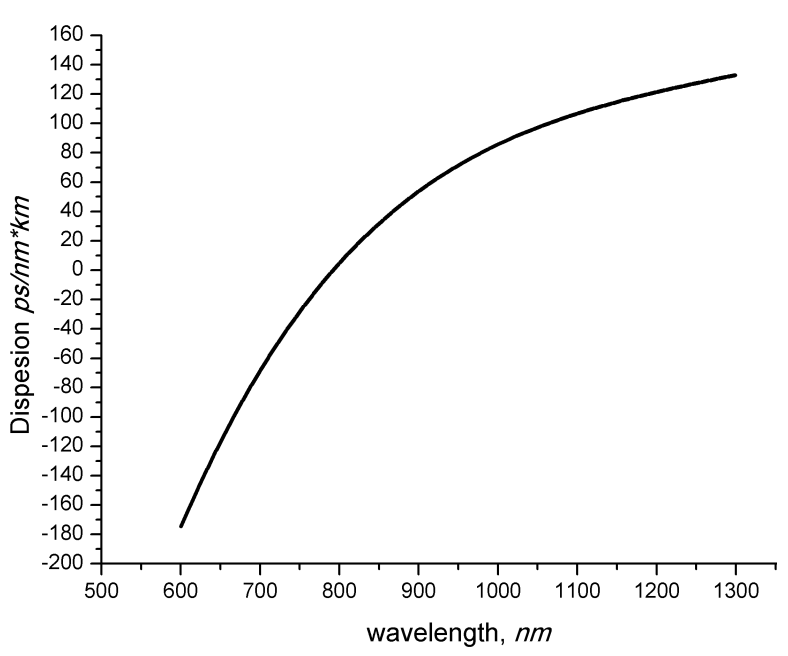

FIG. 2. Dispersion of PCF model

\section{Evaluation of SFWM modes}

The process of spontaneous four-wave mixing (SFWM) at the quantum level can be represented as the virtual absorption of two pump photons $(p)$ with the further generation of pair of photons known as signal $(s)$ and idler $(i)$ photons [1]. Because of the negligibly small response time for Kerr nonlinearity $\chi^{(3)}$, it is possible to detect one photon in the pair in order to notify of the presence of the second one. The signal and idler wavelengths in the SFWM mode for the desired fiber structure and specific laser source pump are determined by the conditions of phase matching and conservation of the energy, see equations (1) and (4):

$$
2 w_{p}=w_{s}+w_{i} .
$$

Nonlinear interaction occurs in the solid silica core of PCFs used for SFWM generation of photon pairs, and the cladding has periodical structure, that consists of air-filled holes.

Two-photon state, caused by four-wave mixing in the optical fiber of the length $L$ can be described by the following expression $[3,4]$ :

$$
|\Psi\rangle=|0\rangle_{s}|0\rangle_{i}+\kappa \iint d w_{s} d w_{i} F\left(w_{s}, w_{i}\right)\left|w_{s}\right\rangle_{s}\left|w_{i}\right\rangle,
$$


where $\kappa$ is a constant, which determines the efficiency of generation. This constant has a linear dependence on the fiber's length, the amplitude of electrical field for each of the pump fields and depends on relative polarization pump fields and generated photon pairs. $F\left(w_{s}, w_{i}\right)$ is the function of spectral amplitude of the biphoton (signal and idler photon), which describes spectral and correlation properties of the generated photon pair. The joint spectral amplitude function can be described as follows:

$$
F\left(w_{s}, w_{i}\right)=\int d w^{\prime} \alpha_{1}\left(w^{\prime}\right) \alpha_{2}\left(w_{s}+w_{i}-w^{\prime}\right) \times \sin c\left[\frac{L}{2} \Delta k\left(w^{\prime}, w_{s}, w_{i}\right)\right] \exp \left[i \frac{L}{2} \Delta k\left(w^{\prime}, w_{s}, w_{i}\right)\right],
$$

where $\alpha_{1,2}(w)$ is spectral pump amplitudes; $\Delta k\left(w^{\prime}, w_{s}, w_{i}\right)$ is the function of phase matching, that for the case of two-photon pump and one polarization for signal and idler fields, is given by the equation:

$$
\begin{gathered}
\Delta k\left(w_{p}, w_{s}, w_{i}\right)=k\left(w_{p}\right)+k\left(w_{s}+w_{i}-w_{p}\right)-k\left(w_{s}\right)-k\left(w_{i}\right)-\left(\gamma_{1} P_{1}+\gamma_{2} P_{2}\right), \\
k_{q}=\frac{\omega_{q} n^{e f f}\left(\omega_{q}\right)}{c}, \quad q=p, s, i,
\end{gathered}
$$

where $\gamma_{1} P_{1}+\gamma_{2} P_{2}$ is the self-cross phase modulation for two pumps with peak powers $P_{1}$ and $P_{2}$ and corresponds to the nonlinearity coefficients $\gamma_{1}$ and $\gamma_{2}$, which in their turn depend on the fitted fiber and pump wavelength, and is taken into account [6,7]. Limitations of energy conservation are determined by the second term of phase detuning in equation (3). The factorable state is the state, where $F\left(w_{s}, w_{i}\right)$ is the result of multiplication of two functions: eigen-functions of signal, $S\left(w_{s}\right)$, and idler, $I\left(w_{i}\right)$, photons.

The results of numerical simulations for the effective index of refraction and PCF dispersion allowed us to obtain the matching conditions and the joint spectral amplitude for the signal and idler photons. The objective of the simulation is to find the modes of generation of photon pairs with minimum spectral correlation and wavelengths commonly used for the telecommunications applications. Calculations of the joint spectral amplitudes at different wavelengths, based on the commercial PCFs models and laboratory femtosecond facilities, were performed. In this sense, the most promising type of PCF was found (Fig. 2). The joint spectral amplitude of eigen-function for the PCF is shown in Fig. 3 and it demonstrates the probability for the generation of signal ( $X$-axis) and idler ( $Y$-axis) photons.

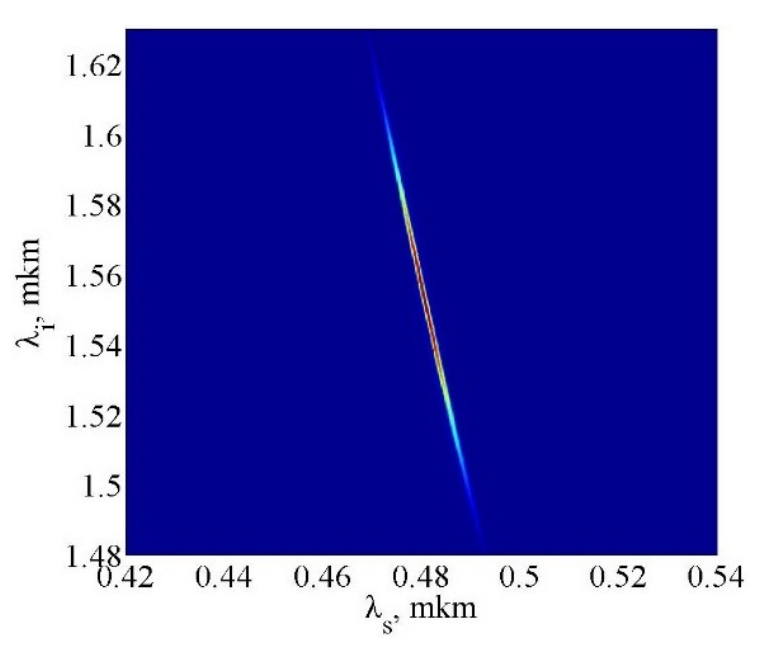

(a)

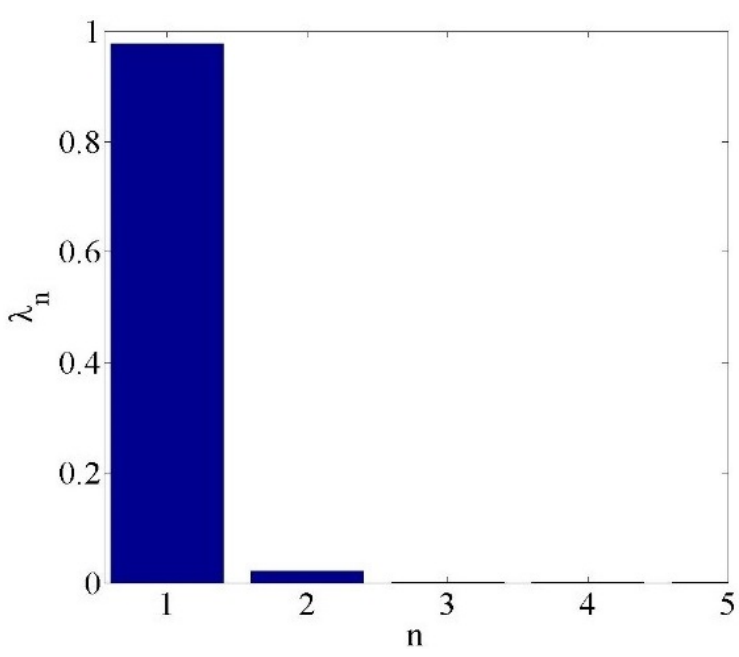

(b)

FIG. 3. Joint spectral amplitude for the signal and idler photons (a) and Schmidt decomposition for PCF (800 nm zero dispersion) with $734 \mathrm{~nm}$ pump wavelength (b)

In order to determine the purity of single-photon states, a method for the decomposition of the spectral amplitude function of two-photon states $F$ into Schmidt modes was developed [7]. Assume that initial state vector can be described as follows:

$$
|\psi\rangle=|0\rangle+\iint F\left(\omega_{i}, \omega_{s}\right) a^{+}\left(\omega_{i}\right) a^{+}\left(\omega_{s}\right) d \omega_{i} d \omega_{s}
$$

Therefore, Schmidt decomposition is given by:

$$
F\left(\omega_{i}, \omega_{s}\right)=\sum_{n} \sqrt{\lambda_{n}} \alpha_{n}\left(\omega_{i}\right) \beta_{n}\left(\omega_{s}\right), \quad \sum_{n} \lambda_{n}=1,
$$


where $\alpha_{n}\left(\omega_{i}\right)$ and $\beta_{n}\left(\omega_{i}\right)$ are Schmidt functions (modes), and $\lambda_{n}$ are decomposition coefficients by Schmidt modes [7]. For the case of pure (factorable) states in the Schmidt decomposition, there is only one mode, wherein the Schmidt number equals 1. Therefore, evaluation of the Schmidt number for SFWM generation mode provides the estimation of purity for single-photon state. Conducted optimization using the parameters of laser pump allowed us to find the most promising mode to generate photons with small spectral correlation, when Schmidt number equals 1.0451 , at $734 \mathrm{~nm}$ pump for wavelengths of 1550 and $480 \mathrm{~nm}$.

\section{Conclusion}

This study includes results from the analysis of PCF structures similar to commercial PCFs in order to generate correlated photon pairs within the process of spontaneous four-wave mixing with minimum spectrum correlation at frequently used telecommunications wavelengths. Numerical simulation of these structures was performed and the dependence of effective index of refraction and dispersion of wavelength was found. Based on the obtained results, promising modes were found to generate single photons with small spectrum correlation for the following conditions: hexagonal structure (Fig. 1), zero dispersion wavelength $800 \mathrm{~nm}, 734 \mathrm{~nm}$ pump, Schmidt number of 1.0451 and wavelength of $1550 \mathrm{~nm}$.

\section{Acknowledgements}

The reported study was funded by RFBR according to the research projects No. 16-02-00972, No. 16-32-60054 mol_a_dk, No. 16-32-00397 mol_a.

\section{References}

[1] Migdall A., Polyakov S.V., Fan J., Bienfang J.C. Single-Photon Generation and detection. Experimental Methods in the Physical Sciences. Elsevier, Oxford, 2013, $624 \mathrm{p}$.

[2] Garay-Palmett K. et al. Photon pair-state preparation with tailored spectral properties by spontaneous four-wave mixing in photonic-crystal fiber. Optics Express, 2007, 15 (22), P. 14870-14886.

[3] Mandel F., Wolf E. Optical Coherence and Quantum Optics. Cambridge University Press, Cambridge, 1995,1166 p.

[4] Chen J., Li X., Kumar P. Two-photon-state generation via four-wave mixing in optical fibers. Phys. Rev. A, $2005,72,033801$.

[5] Agrawal G.P. Nonlinear Fiber Optics, 4th Ed. Elsevier, Oxford, 2007, 552 p.

[6] McKinstrie C.J., Kogelnik H., Schenato L. Four-wave mixing in a rapidly-spun fiber. International Journal of Engineering and Advanced Technology, 2014, 15, P. 8516-8534.

[7] Law Sh.C.K., Walmsley I.A., Eberly J.H. Continuous Frequency Entanglement: Effective Finite Hilbert Space and Entropy Control. Phys. Rev. Lett., 2000, 84, P. 5304-5307. 